\title{
Checklist das espécies de Lauxaniidae (Insecta, Diptera) do estado do Mato Grosso do Sul
}

\author{
Vera Cristina Silva
}

\begin{abstract}
Departamento de Morfologia e Fisiologia Animal, Faculdade de Ciências Agrárias e Veterinárias, UNESP- Universidade Estadual Paulista, Via de acesso Prof. Paulo
\end{abstract} Donato Castellane s/n, 14884-900 Jaboticabal, SP. (vcsilva@fcav.unesp.br)

Recebido 21 novembro 2016

Aceito 6 fevereiro 2017

DOI: $10.1590 / 1678-4766 e 2017146$

\begin{abstract}
Checklist of the species of Lauxaniidae (Insecta, Diptera) from the state of Mato Grosso do Sul. Lauxaniidae is one of the largest Diptera Schizophora families, worldwide in distribution, very common in tropical areas. The family comprises about 1,550 species. Adults may be small to moderately large (2-11 mm), varied in color patterns, often with marks, spots, bands, or reticulating patterns. Larval lauxaniids are widely documented as saprophages, feeding in a diverse array of decaying plant matter, and even feeding in flower heads. Adults are fungal grazers on leaves. The Brazilian fauna of lauxaniids comprises at the moment 74 species of which 8 have been recorded from the state of Mato Grosso do Sul.
\end{abstract}

KEYWORDS. Schizophora, biodiversity, species inventory, Biota-MS Program.

RESUMO. Lauxaniidae é uma das maiores famílias de Diptera Schizophora, com distribuição mundial, muito abundante nas áreas tropicais. A família está composta por cerca de 1.550 espécies. Os adultos apresentam o corpo pequeno a relativamente grande (2-11 mm), com coloração variada, muitas vezes com marcas, manchas, listras ou padrões reticulados. As larvas são conhecidas como saprófagas, alimentando-se em uma variedade de matéria vegetal em decomposição e mesmo em capítulos de flores. Adultos são raspadores de fungos em folhas. A fauna brasileira de Lauxaniidae compreende, até o momento, 74 espécies das quais 8 possuem registro assinalado para o estado do Mato Grosso do Sul.

PALAVRAS-CHAVE. Schizophora, biodiversidade, inventário de espécies, Programa Biota-MS.

Os Lauxaniidae constituem uma das maiores famílias de Diptera Schizophora, com distribuição mundial, muito abundantes nas áreas tropicais, e ausentes na região Antártica (KIM, 1994). A família está composta por 149 gêneros e cerca de 1.550 espécies válidas, compreendidas em três subfamílias, Lauxaniinae, Homoneurinae e Eurychoromyiinae (GAIMARI \& SILVA, 2010a), todas ocorrendo no Brasil e apenas Lauxaniinae com representantes conhecidos no estado do Mato Grosso do Sul.

Na região temperada, larvas da maioria das espécies são saprófagas, vivendo em folhas caídas e em outros materiais vegetais em decomposição. No Brasil, pouco é conhecido dos hábitos dos imaturos. Os adultos variam entre 2-11 mm de comprimento, com asas maculadas ou não, poucos ativos e são encontrados em lugares com sombra e úmidos.

A lista de espécies apresentada neste trabalho foi obtida a partir da literatura e do exame de espécimes depositados na coleção entomológica do Museu de Zoologia, Universidade de São Paulo (MZUSP).

\section{RESULTADOS E DISCUSSÃO}

Até o presente foram registrados seis gêneros e oito espécies. Na Tabela I estão elencadas as espécies do Estado de Mato Grosso do Sul catalogadas até junho de 2010.

Tab. I. Lista de espécies de Lauxaniidae (Diptera) do Estado de Mato Grosso do Sul.

\begin{tabular}{l}
\hline Nome científico \\
\hline Lauxaniinae \\
Allominettia xanthiceps (Williston, 1897) \\
Chaetominettia brasiliensis (Walker, 1853) \\
Chaetominettia corollae (Fabricius, 1805) \\
Neogriphoneura tertia Curran, 1942 \\
Physegenua ferruginea Schiner, 1868 \\
Physegenua lineata Curran, 1942 \\
Poecilominettia picticornis (Coquillett, 1904) \\
Scutominettia mallochi Hendel, 1932
\end{tabular}

Comentários sobre a lista, riqueza do estado comparado com outras regiões. $O$ conhecimento da família para o Brasil inclui 74 espécies de lauxaniídeos. Em uma área de floresta estacional semidecidual no estado de São Paulo foram identificadas dez espécies conhecidas e 35 novas em 17 gêneros conhecidos e 5 gêneros novos, implicando em $78,7 \%$ da fauna da família na área constituída de espécies novas. Comparando-se esses dados com aqueles conhecidos até o momento para o Mato Grosso do Sul, a análise do material do projeto SISBIOTA ("Rede temática para estudos de diversidade, sistemática e limites distribucionais de 
Diptera nos estados do Mato Grosso, Mato Grosso do Sul e Rondônia" - CNPq/FAPESP) certamente irá ampliar em muito a fauna conhecida de lauxaniídeos, sendo esta a razão para a projeção de pelo menos 45 espécies no estado.

Principais grupos de pesquisa. O Dr. Hugo de Souza Lopes, do Instituto Oswaldo Cruz no Rio de Janeiro, coletou amplamente exemplares da família em vários locais do Brasil, notadamente no Rio de Janeiro e no Pará, além de ter compilado a bibliografia sobre a família sem, no entanto, ter produzido publicação a respeito.

O grupo de pesquisa em Sistemática e Taxonomia de Lauxaniidae no Brasil está sob minha coordenação (UNESP, Jaboticabal) e conta ainda com a participação do Dr. Ramon Luciano de Mello (UFMS, Campo Grande).

Principais acervos. As principais instituições detentoras de acervo de Lauxaniidae no Brasil, de acordo com os dados levantados por CARvALHO et al. (2002) e dados pessoais resultantes de visitas às instituições nacionais são, em ordem alfabética: Departamento de Zoologia da Universidade Federal do Paraná (DZUP); Instituto Nacional de Pesquisas da Amazônia (INPA); Museu Nacional do Rio de Janeiro (MNRJ) e Museu de Zoologia da Universidade de São Paulo (MZUSP).

Principais lacunas de conhecimento. A contribuição mais ampla que incluiu os lauxaniídeos neotropicais foi publicada por Hendel (1925). Desde então, houve apenas descrições de novos gêneros ou espécies (SHEwELL, 1986, 1989; BroAdHEAD, 1989; PAPP \& Silva, 1995; Silva, 1998, 1999a,b, 2006; Mello \& Silva, 2008a,b), além do capítulo sobre a família no "Manual of Central American Diptera" (GAImARi \& Silva, 2010b).

Para o estado do Mato Grosso do Sul, as únicas referências de lauxaníideos são aquelas advindas de materiais coletados nos séculos XIX e XX, que redundaram em descrições de novas espécies (CURRAN, 1942). Como indicado por BRANDÃo et al. (2006) e SILVA et al. (2009), a região Centro-Oeste do Brasil é uma das áreas menos amostradas para invertebrados em geral, e Diptera em particular. Um maior impulso para o conhecimento taxonômico de Lauxaniidae do estado, sem dúvida, será proporcionado pelo Projeto SISBIOTA (CNPq/FAPESP).

Perspectivas de pesquisa para os próximos 10 anos. O projeto "Rede temática para estudos de diversidade, sistemática e limites distribucionais de Diptera nos estados do Mato Grosso, Mato Grosso do Sul e Rondônia", aprovado no âmbito do Edital SISBIOTA, em uma parceria do CNPq com a FAPESP abriu grande perspectiva nos estudos sobre a ordem Diptera, particularmente Lauxaniidae no Brasil. O projeto teve início em fevereiro de 2010 e vem amostrando, de forma continuada, localidades estratégicas nesses três estados. Desse modo, espera-se que haja um aumento significativo no conhecimento da diversidade de dípteros, incluindo Lauxaniidae, nos próximos anos.

Agradecimentos. A Fundação de Apoio ao Desenvolvimento do Ensino, Ciências e Tecnologia do Estado de Mato Grosso do Sul (Fundect) e a Superintendência de Ciências e Tecnologia do Estado de Mato Grosso do Sul (Sucitec/MS) pelo convite de participação neste fascículo especial da Iheringia, Série Zoologia e o suporte financeiro para sua publicação. Ao Conselho Nacional de Desenvolvimento Científico e Tecnológico (CNPq) (Proc. 563256/2010-9) e Fundação de Amparo à Pesquisa do Estado de São Paulo (FAPESP) (Proc. 2010/52314-0) pelo auxílio conferido ao projeto "Rede temática para estudos de diversidade, sistemática e limites distribucionais de Diptera nos estados do Mato Grosso, Mato Grosso do Sul e Rondônia", aprovado no âmbito do Edital SISBIOTA.

\section{REFERÊNCIAS BIBLIOGRÁFICAS}

Brandão, C. R. F.; Cancello, E. M.; Yamamoto, C. I. \& Scott-Santos, C. 2006. Perfil do Conhecimento da Diversidade de Invertebrados Terrestres no Brasil. In: Levinsohn, T. org. Avaliação do Estado do Conhecimento da Biodiversidade Brasileira. Brasília, Ministério do Meio Ambiente. Série Biodiversidade vol. 15, p. 205-259.

Broadhead, E. C. 1989. The species of Poecilominettia, Homoeominettia and Floriminettia (Diptera, Lauxaniidae) in Panama. Bulletin of the British Museum (Natural History), Entomology series 58(2):185-226.

Carvalho, C. J. B.; Couri, M. S.; Toma, R.; Rafael, J. A.; Harada, A. Y.; Bonatto, S. R.; Henriques, A. L. \& Gastal, H. A. O. 2002. Principais Coleções Brasileiras de Diptera. Histórico e Situação Atual. In: Costa, C.; Vanin, S. A.; Lobo, J. M. \& Melic, A. orgs. Proyecto de Red Iberoamericana de Biogeografía y Entomología Sistemática. Zaragoza, PRIBES, Sociedad Entomológica Aragonesa (SEA) \& Cyted. vol. 2, p. 37-52.

Curran, C. H. 1942. American Diptera. Bulletin of the American Museum of Natural History 80:51-84.

Gaimari, S. \& Silva, V. C. 2010a. Revision of the Neotropical subfamily Eurychoromyiinae (Diptera: Lauxaniidae). Zootaxa 2342:1-64.

Gaimari, S. \& Silva, V. C. 2010b. 71. Lauxaniidae. In: Brown, B. V.; Borkent, A.; Cumming, J. M.; Wood, D. M.; Woodley, N. E. \& Zumbado, M. A. eds. Manual of Diptera of Central America. Ottawa, NRC Press. vol. 2, p. 971-995.

HENDEL, F. 1925. Neue Ubersicht über die bisher bekannt gewordenen Gattungen der Lauxaniidae, nebst Beschreibung neuer Gattungen und Arten. Encyclopedie entomologique, Serie B, II Diptera. p.103-142.

KIM, S. P. 1994. Australian lauxaniid flies: revision of the Australian species of Homoneura van der Wulp, Trypetisoma Malloch, and allied genera (Diptera: Lauxaniidae). Victoria, Australia CSIRO. 445p.

Mello, R. J. C. L. \& Silva, V. C. 2008a. Revision of the genus Physoclypeus Hendel, 1907 (Diptera, Lauxaniidae), with description of seven new species. Papéis Avulsos de Zoologia 48:289-315.

Mello, R. J. C. L. \& Silva, V. C. 2008b. A taxonomic review of Neogriphoneura Malloch, 1924 (Diptera: Lauxaniidae), with description of three new species. Zootaxa 1806:35-46.

PAPP, L. \& Silva, V. C. 1995. Seven new genera of the Neotropical Lauxaniidae (Diptera). Acta Zoologica Hungarica 41(3):185-208.

Shewell, G. E. 1986. New American genera of Lauxaniidae, based on species of earlier authors, and a note on Lyciella rorida (Fallén) in North America (Diptera). Canadian Entomologist 118:537-547.

Shewell, G. E. 1989. Sapromyza lopesi sp. n. from Brazil: a species related to $S$. duodecimvittata (Frey, 1919) (Diptera: Lauxaniidae). Memórias do Instituto Oswaldo Cruz 84(Supl. IV):483-485.

SiLva, V. C. 1998. Systematic review of Neotropical Lauxaniidae genera: Triconopsis Hendel 1914 (Diptera, Schizophora). Revista Brasileira de Zoologia 15(3):783-788.

SILVA, V. C. 1999a. Systematic review of Neotropical Lauxaniidae genera: Cephalella Malloch, 1926 (Diptera, Schizophora). Revista Brasileira de Zoologia 16(1):133-137.

SILVA, V. C. 1999b. Four new species of Bacilloflagellomera Papp \& Silva, 1995 (Diptera, Lauxaniidae). Deutsche Entomolgische Zeitschrift 46(1):109-118.

SiLvA, V. C. 2006. Systematic review of Neotropical genera of Lauxaniidae: 3. The genus Paracestrotus Hendel, 1925 (Diptera: Schizophora). Zootaxa 1249:61-68.

Silva, V. C.; Lamas, C. J. E.; Amorim, D. S. \& Nihei, S. S. 2009. Diptera. In: RochA, R. M DA \& BoEGER, W. A. P. eds. Estado da arte e perspectivas para a Zoologia no Brasil. Curitiba, Editora Universidade Federal do Paraná, p. 157-166. 